\title{
¿Movilización selectiva del conocimiento a través de los blogs? el caso de las experiencias de aprendizaje-servicio en las universidades españolas
}

\author{
María R. Belando-Montoro \& María Aranzazu \\ Carrasco Temiño
}

\begin{abstract}
Resumen
Este artículo parte de la necesidad cada vez mayor de movilizar el conocimiento de modo que pueda ser útil para toda la sociedad. En este marco, el estudio presentado se centra en la movilización del conocimiento generado por las experiencias de aprendizaje-servicio (ApS) desarrolladas a través de proyectos en diversas universidades españolas. Para ello, el objetivo planteado fue seleccionar y analizar los blogs a través de los cuales se difunden experiencias y proyectos universitarios de ApS realizados en España. La metodología utilizada se basa en la selección, observación y recogida de datos homogéneos que permitan una posterior comparación entre ellos para facilitar la detección de las tendencias más seguidas por los blogs. El estudio revela la escasa información y el limitado conocimiento que se moviliza a través de este medio, por lo que se ofrecen una serie de sugerencias para,en primer lugar, el aumento de la movilización del conocimiento generado en los proyectos de ApS que se realizan en el ámbito universitario y, en segundo lugar, la mejora de la visibilidad de los blogs construidos para ello. Estas sugerencias están relacionadas con la estructura y la situación del blog y con el uso de redes sociales.
\end{abstract}

Palabras clave:

aprendizaje-servicio; blogs; universidad; difusión del conocimiento científico 


\title{
Selective knowledge mobilization through the blogs? The case of service- learning experiences at the spanish university
}

\begin{abstract}
This article is based on the growing need to mobilize knowledge so that it can be useful for the whole society. In this context, the study presented focuses on the mobilization of knowledge generated by servicelearning experiences developed through projects in various Spanish universities. To this end, the objective was to select and analyze the blogs through which university-based learning-service experiences and projects in Spain are disseminated. The methodology used is based on the selection, observation and collection of homogeneous data that allow a subsequent comparison between them to facilitate the detection of the trends most followed by blogs. The study reveals the scarce information and the limited knowledge that is mobilized through this medium, so a series of suggestions are offered for, firstly, the increase of the mobilization of the knowledge generated in the projects of Service-Learning that are realized in the university environment and, secondly, improving the visibility of blogs built for it. These suggestions are related to the structure and the situation of the blog and with the use of social networks.
\end{abstract}

Keywords: service-learning; blogs; college; diffusion of scientific knowledge

\section{Mobilização seletiva do conhecimento através dos blogues? O caso das experiências de aprendizagem-serviço nas universidades espanholas}

\begin{abstract}
Resumo: Este artigo parte da necessidade crescente de mobilizar o conhecimento de modo a que ele possa ser útil para toda a sociedade. Neste contexto, o estudo apresentado centra-se na mobilização do conhecimento gerado pelas experiências de aprendizagem-serviço (ApS) desenvolvidas através de projetos em várias universidades espanholas. Assim, o objetivo estabelecido foi selecionar e analisar blogues através dos quais se difundem experiências e projetos universitários de ApS realizados em Espanha. A metodologia utilizada baseiase na seleção, observação e recolha de dados homogéneos que permitam uma posterior comparação entre eles para facilitar a deteção das tendências mais seguidas pelos blogues. O estudo revela a escassa informação e o limitado conhecimento que se mobiliza através deste meio, pelo que se apresentam diversas sugestões para, em primeiro lugar, aumentar a mobilização do conhecimento gerado nos projetos de ApS que se realizam a nível universitário e, em segundo lugar, melhorar a visibilidade de blogues construídos para isso. Estas sugestões estão relacionadas com a estrutura e situação do blogue e com o uso de redes sociais.
\end{abstract}

Palavras-chave: aprendizagem-serviço; blogues; universidade; difusão do conhecimento científico

\section{¿Mobilisation selectivedes connaissances par le blogs? Le cas d'experiences d'apprentissage par le service dans l'université espagnole}

Résumé: Cet article est basé sur la nécessité de plus en plus grande de mobiliser les connaissances afin que celles-ci puissent être utiles pour la société. Dans ce contexte, l'étude présentée porte sur la mobilisation des connaissances générées par les expériences de service-learning développées grâce à des projets dans diverses universités espagnoles. Pour cela, l'objectif déclaré était de sélectionner et d'analyser des blogs à travers lesquels des expériences et des projets d'apprentissage du service universitaire menés en Espagne sont diffusés. La méthodologie utilisée est basée sur la sélection, l'observation et la collecte de données homogènes pour permettre une comparaison ultérieure entre eux pour faciliter la détection des tendances suivies par la plupart des blogs. L'étude révèle des informations limitées et une connaissance limitée à mobiliser par ce moyen, donc on offre un certain nombre de suggestions pour, d'abord, une mobilisation accrue des connaissances générées dans les projets d'apprentissage par le service effectué au niveau de l'université et, d'autre part, l'amélioration de la visibilité des blogs construits pour elle. Ces suggestions sont liées à la structure et au statut du blog et à l'usage des réseaux sociaux.

Mots-clés: apprentissage par le service; blogs; université; diffusion des connaissances scientifiques 


\section{Introducción}

La educación superior tiene un gran reto frente a este mundo global y plural en el que vivimos. La formación para la vida en el siglo XXI y la preparación para el ámbito laboral deben abordarse desde una dimensión social que ponga el acento en la ciudadanía activa y responsable. Las generaciones más jóvenes deben adquirir las competencias necesarias para vivir de manera sostenible, no sólo a nivel personal sino también laboral.

En el marco de estos propósitos, las universidades a través de la formulación de objetivos y competencias transversales en los diversos planes docentes han pretendido garantizar esta formación. Sin embargo, la implicación docente no ha acompañado este esfuerzo, quizás debido algunas veces a mero desconocimiento de cómo integrarlo en sus prácticas y, otras veces, por la saturación de responsabilidades y funciones a las que se ve sometido el profesorado, especialmente en los últimos años. Consecuentemente, la realidad muestra que a pesar de la presencia de referencias a la ciudadanía activa y al desarrollo de valores sociales en los programas de asignaturas de diferentes titulaciones, no se llevan a la práctica suficientes actividades académicas que permitan la adquisición de estas competencias.

Las propuestas de aprendizaje-servicio en la universidad se presentan como una opción viable que permiten dar respuesta a estas lagunas ya que, como sostiene Martínez $(2010,16)$,

"Tanto los fundamentos pedagógicos, sociales y éticos que caracterizan el APS como el sentido de cooperacióny colaboración que cultiva en el estudiante y que promueve como servicioen la comunidad hacen de las propuestas de APS un buen espacio deaprendizaje de competencias para la práctica profesional y también potencialmentepara la práctica de ciudadanía activa".

El aprendizaje-servicio es además una metodología educativa eficaz para el desarrollo sostenible y, al mismo tiempo, es intrínsecamente sostenible ya que a través de ella se desarrollan trabajospor la sostenibilidad social y medioambiental (Aramburuzabala, Cerrillo y Tello, 2015:79). Rodríguez-Gallego $(2014,97)$ sintetiza sus beneficios en los siguientes ámbitos: currículum académico, formación en valores y vinculación con la comunidad.

El Aprendizaje Servicio (ApS) es, por tanto, una herramienta educativa de gran valor que en los últimos años está adquiriendo poco a poco un mayor protagonismo. Este desarrollo es lento en el ámbito universitario. Como se aprecia en la Red Universitaria Española de Aprendizaje-Servicio (ApS-U), las iniciativas y proyectos desarrollados en 
España son todavía escasos. No obstante, en los últimos años se han publicado diversas obras colectivas en las que se reflexiona sobre su marco teórico y se describen diferentes experiencias realizadas desde el ámbito universitario, como la de Martínez (ed.) (2010), lade Rubio, Prats y Gómez (coord.) (2013), la de Santos, Sotelino y Lorenzo (2015)y la de Deeley (2016), entre otras. Asimismo se han multiplicado los artículos en revistas científicas que exponen las diversas virtudes de esta metodología y algunas han llegado a dedicar monográficos a esta temática. Véase como ejemplo el monográfico de Profesorado. Revista de currículum y formación del profesorado en 2015, el de la Revista Internacional de educación para la justicia social en 2013 y el de la revista Cuadernos de Pedagogía en 2006. Es también de interés la creación, en el año 2015, de la Revista Iberoamericana de Aprendizaje Servicio por parte de la Red Iberoamericana de Aprendizaje Servicio y la Red Universitaria Española de Aprendizaje Servicio.

Ahora bien, las experiencias desarrolladas necesitan una difusión más allá de las publicaciones académicas que limitan enormemente su potencial impacto. Una herramienta útil para la difusión educativa, y que pueden ser de gran ayuda para la aplicación práctica del conocimiento generado en proyectos desarrollados y financiados mayormente en convocatorias públicas, son los blogs. A través de éstos se puede difundir, comunicar y movilizar el conocimiento generado en las experiencias y proyectos de aprendizaje-servicio y alcanzar una mayor visibilidad.

Este estudio se centra, por ello, en seleccionar y analizar los blogs a través de los cuales se difunden experiencias y proyectos universitarios de aprendizaje-servicio realizados en España, con el objetivo de extraer la información publicada, analizarla y realizar propuestas que puedan mejorar la difusión de dichas experiencias así como el conocimiento generado a través de éstas.

\section{Metodología}

La metodología utilizada para el análisis de los blogs sobre experiencias y proyectos de Aprendizaje-Servicio se basa en la selección, observación y recogida de datos homogéneos que permitan una posterior comparación entre ellos para facilitar la detección de las tendencias más seguidas por los blogs.

La observación que se realiza en el medio virtual es similar a la realizada en entornos convencionales, aunque puede llegar a adoptar un mayor dinamismo e interacción (Orellana y Sánchez, 2006:211). Sin embargo, una de sus desventajas es la falta de definición de los límites del campo a estudiar.

Los blogs se utilizan actualmente para un amplio espectro de funciones. Según Torres-Salinas y Cabezas-Clavijo (2008) existen cuatro usos básicos de los blogs:

- Medio de publicación sin intermediarios.

- Tablón de anuncios y repositorio personal o colectivo. 
- Como medio de difusión selectiva de la información.

- Acercar la ciencia al público no especializado.

Centrándonos en el uso de los blogs como medio de difusión de experiencias de Aprendizaje-Servicio, éstos se encuentran principalmente enmarcados en un uso de los blogs como tablón de anuncios y repositorio personal o colectivo ya que, tal y como describe este uso, los blogs "se convierten [...] en una plataforma atractiva para aumentar la visibilidad y el impacto de los contenidos" (Torres-Salinas y Cabezas-Clavijo, 2008:3). Pero, de algún modo y como se mostrará más adelante, también se utilizan para los otros tres usos. Los blogs suponen un medio de publicación sin intermediarios, por lo que se publica la información que se quiere o como se quiere, sin estar sometido a ningún criterio o evaluación externa. Evidentemente, también se pretende en la mayoría de los casos acercar la ciencia, en este caso, los proyectos realizados sobre aprendizaje-servicio desde la universidad al público no especializado y tener así un mayor impacto. Por último, y quizá a consecuencia de todo lo anterior, supone un medio en el que se difunde una selección de la información de la que se dispone y se moviliza el conocimiento generado.

Debemos tener presente, por tanto, que el análisis de los blogs se basa en su carácter de medio de difusión de experiencias llevadas a cabo, su proceso y conclusiones. La cuestión será comprobar qué parte del proceso y de las conclusiones se difunde.

Atendiendo a estas característicasy usos de los blogs, el desarrollo de la metodología de recogida de datos se ha centrado en la observación virtual de dichos blogs. Garrido (2003) realiza una definición muy acertada de la observación que hemos realizado en este estudio, ya que define la observación periférica como una actuación desde un participante pasivo o lurker, que nos permite acceder a la prá lar a la realizada en entornos convencionales, aunque puede llegar a adoptar un mayor dinamismo e intáctica sin intervenir en su desarrollo. Este tipo de metodología permite realizar un análisis de tipo descriptivo, sociotécnicoy discursivo de los blogs a trabajar.

\section{Selección de la muestra}

Para seleccionar la muestra objeto de análisis, todas las experiencias de ApSdebían haber sido realizadas en España y cumplir con una serie de requisitos que nos permitieran realizar una comparativa homogénea entre los blogs. En concreto, la experiencia o proyecto de ApS:

- Tiene un blog en el que ofrece detalles del proceso, especialmente, incluye objetivos, metodología y evaluación.

- Proviene de una iniciativa universitaria, por lo tanto, uno de los grupos participantes son estudiantes universitarios. 
Tras realizar una primera búsqueda de blogs donde se recogen actividades/proyectos de Aprendizaje-Servicio, se seleccionan para la muestra 8 de estos blogs, los cuales cumplen con los requisitos indicados. La búsqueda se realizó inicialmente a través de la Red Española de Aprendizaje-Servicio pero fue necesario, asimismo, utilizar el buscador de Google.

No se utilizó ningún requisito temporal para la selección. Todos los blogs que constituyen la muestra se han elaborado en un periodo relativamente corto: entre los años 2005 al 2012. No se han encontrado blogs anteriores ni más recientes.

Los blogs que constituyen la muestra de este estudio son los siguientes: Taller Comunica, Proyecto ADAMS, Proyecto PEINAS, Amigos y Amigas de la Lectura, Jardín Miguel Martí i Pol, Proyecto SOU-esTUtutor, Safalud y JovexJove.

\section{Análisis de la muestra por categorías}

Para el análisis de los 8 blogs de ApS se han elaborado dos tablas a partir de una serie de categorías que nos permiten comparar los datos fácilmente e identificar las similitudes y las diferencias.

Las categorías por las que se han estructurado las tablas siguientes ("Fig. 1 y 3") siguen una estructura basada en la definición y características principales que debe tener la experiencia/proyecto deAps:

- Nombre de la experiencia

- Ámbito/Sector

- Sujetos 1

- Sujetos 2

- Descripción

- Universidad/Facultad

- Objetivos para sujetos 1

- Objetivos para sujetos 2

- Temporalidad

- Lugar

- Evaluación

Además, se incluye una tabla ("Fig.4") donde se incluyen los blogs y los enlaces de cada una de las experiencias de ApS que describimos.

\section{Resultados}

Los resultados del análisis de las diferentes experiencias de ApS seleccionadas se presentan principalmente a través de las figuras 1 y 3 donde se recoge la información 
relativa a las diferentes categorías de las experiencias de Aprendizaje-Servicio realizadas desde universidades españolas.

En la figura 1 se incluye el ámbito/sector, sujetos 1 (los que llevan a cabo las diferentes actividades), sujetos 2 (a los que van dirigidas las actividades de ApS), una breve descripción de la experiencia y la Facultad y Universidad desde la que parte la iniciativa de la experiencia.

FIGURA 1: EXPERIENCIAS APS I

\begin{tabular}{|c|c|c|c|c|c|}
\hline Nombre & $\begin{array}{l}\text { Ámbito/ } \\
\text { Sector }\end{array}$ & Sujetos 1 & Sujetos 2 & Descripción & $\begin{array}{l}\text { Universidad/ } \\
\text { Facultad }\end{array}$ \\
\hline $\begin{array}{c}\text { Taller } \\
\text { Comunica }\end{array}$ & $\begin{array}{l}\text { Educación } \\
\text { formal }\end{array}$ & $\begin{array}{l}\text { Estudiantes } \\
\text { ESADE (ADE) }\end{array}$ & $\begin{array}{l}\text { Estudiantes } \\
\text { de } \\
\text { secundaria } \\
\text { o jóvenes de } \\
\text { entidades } \\
\text { sociales }\end{array}$ & $\begin{array}{l}\text { Alumnos de ESADE, } \\
\text { con una asignatura } \\
\text { sobre comunicación } \\
\text { en público, } \\
\text { ayudan a alumnos } \\
\text { de segundaria } \\
\text { a preparar una } \\
\text { exposición oral. }\end{array}$ & $\begin{array}{c}\text { ESADE } \\
\text { Administración } \\
\text { y dirección de } \\
\text { empresas }\end{array}$ \\
\hline $\begin{array}{l}\text { Proyecto } \\
\text { ADAMS }\end{array}$ & Hospitalario & $\begin{array}{l}\text { Estudiantes } \\
\text { de Medicina y } \\
\text { Enfermería }\end{array}$ & $\begin{array}{l}\text { Niños } \\
\text { ingresados en } \\
\text { una unidad } \\
\text { de oncología. }\end{array}$ & $\begin{array}{l}\text { Actuaciones como } \\
\text { payasos, magia y } \\
\text { cuentacuentos de } \\
\text { los estudiantes para } \\
\text { los niños ingresados } \\
\text { en la unidad de } \\
\text { oncología }\end{array}$ & $\begin{array}{c}\text { Facultad de } \\
\text { Medicina/ } \\
\text { Universidad del País } \\
\text { Vasco }\end{array}$ \\
\hline $\begin{array}{l}\text { Proyecto } \\
\text { PEINAS }\end{array}$ & Inmigración & $\begin{array}{l}\text { Estudiantes } \\
\text { de Pedagogía } \\
\text { Intercultural } \\
\text { del Grado de } \\
\text { Pedagogía }\end{array}$ & $\begin{array}{c}\text { Diferentes } \\
\text { comunidades } \\
\text { culturales de } \\
\text { Galicia }\end{array}$ & $\begin{array}{l}\text { Se busca que } \\
\text { los alumnos de } \\
\text { la universidad } \\
\text { se acerquen a } \\
\text { diferentes contextos } \\
\text { socioculturales que } \\
\text { les permitan mejorar } \\
\text { sus habilidades y } \\
\text { generen un beneficio } \\
\text { para estos contextos. }\end{array}$ & $\begin{array}{l}\text { Facultad de } \\
\text { Ciencias de } \\
\text { laEducación/ } \\
\text { Universidad de } \\
\text { Santiago de } \\
\text { Compostela }\end{array}$ \\
\hline $\begin{array}{l}\text { Amigos y } \\
\text { amigas de } \\
\text { la lectura }\end{array}$ & $\begin{array}{l}\text { Educación } \\
\text { formal }\end{array}$ & $\begin{array}{c}\text { Estudiantes } \\
\text { de Pedagogía } \\
\text { y Educación } \\
\text { Social }\end{array}$ & $\begin{array}{l}\text { Alumnos de } \\
\text { primaria y } \\
\text { secundaria }\end{array}$ & $\begin{array}{c}\text { Se busca mejorar } \\
\text { las competencias } \\
\text { lectoras, } \\
\text { comprensión oral y } \\
\text { escrita de alumnos } \\
\text { con bajos resultados } \\
\text { académicos. }\end{array}$ & $\begin{array}{l}\text { Facultad de } \\
\text { Educación/ } \\
\text { Universidad de } \\
\text { Barcelona }\end{array}$ \\
\hline $\begin{array}{c}\text { Jardín } \\
\text { Miguel } \\
\text { Martí i Pol }\end{array}$ & Comunidad & $\begin{array}{l}\text { Estudiantes } \\
\text { de Terapia } \\
\text { Ocupacional }\end{array}$ & $\begin{array}{c}\text { Personas con } \\
\text { enfermedad } \\
\text { mental }\end{array}$ & $\begin{array}{l}\text { El proyecto ha } \\
\text { consistido en crear } \\
\text { y mantener un jardín } \\
\text { abierto para toda la } \\
\text { sociedad y dedicado } \\
\text { al poeta por el que } \\
\text { lleva el nombre. }\end{array}$ & $\begin{array}{c}\text { Facultat de } \\
\text { Ciències de la } \\
\text { Salut i el Benestar/ } \\
\text { Universidad de Vic }\end{array}$ \\
\hline
\end{tabular}




\begin{tabular}{|c|c|c|c|c|c|}
\hline Nombre & $\begin{array}{l}\text { Ámbito/ } \\
\text { Sector }\end{array}$ & Sujetos 1 & Sujetos 2 & Descripción & $\begin{array}{l}\text { Universidad/ } \\
\text { Facultad }\end{array}$ \\
\hline $\begin{array}{l}\text { Proyecto } \\
\text { SOU- } \\
\text { esTUtutor }\end{array}$ & Universidad & $\begin{array}{l}\text { Estudiantes de } \\
\text { último año de } \\
\text { carrera }\end{array}$ & $\begin{array}{l}\text { Estudiantes } \\
\text { de primer año } \\
\text { de carrera }\end{array}$ & $\begin{array}{c}\text { Es un programa } \\
\text { de mentoría entre } \\
\text { compañeros } \\
\text { universitarios. Los } \\
\text { estudiantes de último } \\
\text { año de carrera, o ya } \\
\text { finalizada, colaboran } \\
\text { con estudiantes } \\
\text { que ingresan en la } \\
\text { universidad. }\end{array}$ & $\begin{array}{c}\text { Facultad de } \\
\text { Educación/ } \\
\text { Universidad } \\
\text { Complutense de } \\
\text { Madrid }\end{array}$ \\
\hline Safalud & $\begin{array}{l}\text { Educación } \\
\text { formal }\end{array}$ & $\begin{array}{c}\text { Estudiantes } \\
\text { del Ciclo de } \\
\text { Dietética }\end{array}$ & $\begin{array}{l}\text { Estudiantes } \\
\text { del Colegio } \\
\text { Sagrada } \\
\text { Familia }\end{array}$ & $\begin{array}{l}\text { Desarrollar una } \\
\text { participación con los } \\
\text { centros escolares } \\
\text { en los que se } \\
\text { detallen los hábitos } \\
\text { adecuados para una } \\
\text { correcta alimentación } \\
\text { entre los alumnos } \\
\text { de primaria y } \\
\text { secundaria. }\end{array}$ & $\begin{array}{c}\text { Ciclo de Dietética/ } \\
\text { Valladolid }\end{array}$ \\
\hline JovexJove & $\begin{array}{l}\text { Educación } \\
\text { formal }\end{array}$ & $\begin{array}{c}\text { Estudiantes } \\
\text { de } 1^{\circ} \text { de } \\
\text { Educación } \\
\text { Social y } \\
\text { Psicología de } \\
\text { UVic }\end{array}$ & $\begin{array}{l}\text { Estudiantes } \\
\text { de ESO }\end{array}$ & $\begin{array}{l}\text { Apoyar a alumnos } \\
\text { con mayores } \\
\text { dificultades en } \\
\text { sus necesidades } \\
\text { individuales para } \\
\text { lograr una mejora } \\
\text { en su rendimiento a } \\
\text { través de una figura } \\
\text { joven y cercana a los } \\
\text { estudiantes. }\end{array}$ & $\begin{array}{l}\text { Facultatd'Educació, } \\
\text { Traducció i } \\
\text { Ciències Humanes/ } \\
\text { Universidad de Vic. }\end{array}$ \\
\hline
\end{tabular}

Fuente: elaboración propia

Con respecto a las categorías incluidas en la Figura 1 sobre el ámbito, los sujetos protagonistas, la descripción y universidad, podemos observar algunas tendencias en las experiencias seleccionadas.

Por un lado, con respecto al ámbito donde se lleva a cabo el Aprendizaje-Servicio, encontramos un claro protagonismo de la educación formal, aunque en la totalidad de las experiencias se trabaja con poblaciones en riesgo de exclusión o servicios a la comunidad. Este resultado apoya la idea sustentada por Puig, Batlle, Bosch y Palos (2007:20) en su definición sobre el ApS: "es una propuesta educativa que combina procesos de aprendizaje y de servicio a la comunidad en un solo proyecto bien articulado en el que los participantes se forman al trabajar sobre necesidades reales del entorno con el objetivo de mejorarlo". 
En relación con los sujetos pertenecientes a algún ámbito universitario (condición para la selección de la muestra), resulta de interés subrayar que existe una clara tendencia a realizar este tipo de experiencias en las disciplinas de ciencias sociales y de la salud.

FIGURA 2: DISTRIBUCIÓN DE LOS BLOGS DE EXPERIENCIAS APS SEGÚN DISCIPLINA.

\begin{tabular}{|c|c|c|c|c|c|}
\hline & $\begin{array}{c}\text { CC. sociales y } \\
\text { jurídicas }\end{array}$ & $\begin{array}{c}\text { CC. de la } \\
\text { salud }\end{array}$ & $\begin{array}{c}\text { Ingenierías y } \\
\text { arquitecturas }\end{array}$ & $\begin{array}{c}\text { Artes y } \\
\text { humanidades }\end{array}$ & Ciencias \\
\hline $\mathrm{N}^{\mathrm{a}}$ de blogs ApS & 5 & 3 & 0 & 0 & 0 \\
\hline
\end{tabular}

Fuente: elaboración propia

Concretando este dato en los grados universitarios, 4 de los blogs de experiencias ApS se realizan en los grados de Pedagogía, Educación Social y ADE. Entre las experiencias enmarcadas en las Ciencias de la Salud se obtiene una mayor dispersión de las experiencias: Medicina/Enfermería, Terapia Ocupacional y Nutrición y dietética.

Por otro lado, con respecto a los sujetos 2 , indicar que el $50 \%$ de ellos pertenecen a áreas de la educación reglada (Primaria y Secundaria). El restante 50\% de la muestra se distribuye en pacientes oncológicos, inmigrantes, universitarios y personas con enfermedades mentales.

Con respecto ala descripción de las experiencias, como se observa en la figura 1 , se realizan tareas intrínsecamente relacionadas con el grado universitario y nivel educativo de los participantes. En este caso, las actividades están dirigidas a temas transversales, actitudinales o de ocio. Este dato tiene relación con el sentido relacional y de servicio a la comunidad que tiene el ApS.

En la figura 3, que aparece a continuación, se recoge la información relativa a los objetivos de las experiencias, distinguiendo los objetivos 1 (los referidos a los que llevan a cabo las diferentes actividades) de los objetivos 2 (los de aquellos a los que van dirigidas las actividades de ApS), la duración, el lugar en el que se realiza y las técnicas y criterios de evaluación. 
FIGURA 3: EXPERIENCIAS APS II

\begin{tabular}{|c|c|c|c|c|}
\hline Nombre & Objetivos 1 & Objetivos 2 & Temporalidad & Lugar \\
\hline \multirow[t]{2}{*}{$\begin{array}{c}\text { Taller } \\
\text { Comunica }\end{array}$} & $\begin{array}{l}\text { Desarrollar las } \\
\text { capacidades y habilidades } \\
\text { para hablar en público y } \\
\text { ser capaces de motivar y } \\
\text { gestionar grupos. }\end{array}$ & $\begin{array}{c}\text { Interés y valoración } \\
\text { de la expresión oral } \\
\text { y la comunicación en } \\
\text { público. } \\
\text { Capacidades } \\
\text { comunicativas en } \\
\text { general y expresión } \\
\text { oral, estructuración y } \\
\text { método. } \\
\text { Habilidades sociales y } \\
\text { relacionales. }\end{array}$ & $\begin{array}{l}\text { Se distribuye en el } \\
\text { curso académico } \\
\text { en } 3 \text { sesiones de } 2 \\
\text { horas cada una. } \\
\text { Desde } 2011 / 2012\end{array}$ & $\begin{array}{l}\text { Institutos de } \\
\text { secundaria }\end{array}$ \\
\hline & Evaluación & \multicolumn{3}{|c|}{$\begin{array}{l}\text { Exposición oral de cada taller y presentación de videos. } \\
\text { Presentación de la ficha de planificación y desarrollo del taller } \\
\text { Cuestionario de autoevaluación.Valoración de la experiencia } \\
\text { ApS. }\end{array}$} \\
\hline \multirow[t]{2}{*}{$\begin{array}{l}\text { Proyecto } \\
\text { ADAMS }\end{array}$} & $\begin{array}{l}\text { Comprender situaciones } \\
\text { de vida en hospitales. } \\
\text { Desarrollar habilidades de } \\
\text { empatía, responsabilidad } \\
\text { y competencias } \\
\text { profesionales. }\end{array}$ & $\begin{array}{l}\text { Compensar el grave } \\
\text { impacto que tienen en } \\
\text { los niños su estancia } \\
\text { hospitalaria. }\end{array}$ & $\begin{array}{l}\text { Tiene una duración } \\
\text { de } 6 \text { meses con } \\
\text { visitas cada } \\
\text { semana. Desde } \\
\text { 2011/2012 }\end{array}$ & $\begin{array}{l}\text { Servicio } \\
\text { oncología } \\
\text { pediátrica } \\
\text { del Hospital } \\
\text { de Cruces- } \\
\text { Gurutzeta. }\end{array}$ \\
\hline & Evaluación & \multicolumn{3}{|c|}{$\begin{array}{c}\text { Realizar una memoria de la actividad llevada a cabo en el } \\
\text { hospital. }\end{array}$} \\
\hline \multirow[t]{2}{*}{$\begin{array}{l}\text { Proyecto } \\
\text { PEINAS }\end{array}$} & $\begin{array}{l}\text { Conocer genérico de las } \\
\text { culturas y pueblos. } \\
\text { Desarrollar habilidades } \\
\text { personales, relacionales, } \\
\text { sociales y cívicas. }\end{array}$ & $\begin{array}{l}\text { Según la actividad } \\
\text { realizada }\end{array}$ & $\begin{array}{l}\text { Duración de } 6 \\
\text { meses. Desde } 2010\end{array}$ & $\begin{array}{l}\text { Comunidades } \\
\text { culturales de } \\
\text { Galicia }\end{array}$ \\
\hline & Evaluación & \multicolumn{3}{|c|}{$\begin{array}{l}\text { Tutorías, portafolio de la experiencia, que incluye: Objetivos, } \\
\text { ensayo crítico y calificaciones. }\end{array}$} \\
\hline \multirow[t]{2}{*}{$\begin{array}{l}\text { Amigos y } \\
\text { amigas de } \\
\text { la lectura }\end{array}$} & $\begin{array}{l}\text { Acercar al alumno al } \\
\text { mundo profesional con } \\
\text { niños y jóvenes. Mejorar } \\
\text { la paciencia, empatía y } \\
\text { uso de estrategias de } \\
\text { adaptación cada usuario. } \\
\text { Saber hacerse respetar. }\end{array}$ & $\begin{array}{l}\text { Mejorar las } \\
\text { capacidades lectoras } \\
\text { y de comunicación } \\
\text { oral. }\end{array}$ & $\begin{array}{l}\text { En un curso } \\
\text { académico con una } \\
\text { dedicación de dos } \\
\text { horas semanales. } \\
\text { Desde } 2007 / 2008\end{array}$ & $\begin{array}{l}\text { Escuelas e } \\
\text { Institutos de } \\
\text { Barcelona }\end{array}$ \\
\hline & Evaluación & \multicolumn{3}{|c|}{$\begin{array}{c}\text { Se evaluará a través de un diario reflexivo individual, entregas } \\
\text { periódicas, participar en la actividad grupal y asistir a las } \\
\text { sesiones de formación organizadas por la oficina de ApS. }\end{array}$} \\
\hline
\end{tabular}




\begin{tabular}{|c|c|c|c|c|}
\hline Nombre & Objetivos 1 & Objetivos 2 & Temporalidad & Lugar \\
\hline \multirow[t]{2}{*}{$\begin{array}{l}\text { Jardín } \\
\text { Miguel } \\
\text { Martí i Pol }\end{array}$} & $\begin{array}{c}\text { Elaborar diseños y } \\
\text { desarrollar proyectos. } \\
\text { Desarrollar competencias } \\
\text { de la invest. cualitativa. } \\
\text { Desarrollar el rol de } \\
\text { jardinero y Terap. Ocupac. }\end{array}$ & $\begin{array}{c}\text { Empoderar su papel } \\
\text { como participantes en } \\
\text { la comunidad en que } \\
\text { vive. } \\
\text { Reivindicar sus } \\
\text { derechos como } \\
\text { ciudadanos. }\end{array}$ & $\begin{array}{l}\text { Desde } 2005 \text { hasta } \\
\text { la actualidad (por } \\
\text { cursos académicos) }\end{array}$ & Jardín \\
\hline & Evaluación & \multicolumn{3}{|c|}{ No se detalla. } \\
\hline \multirow[t]{2}{*}{$\begin{array}{l}\text { Proyecto } \\
\text { SOU- } \\
\text { esTUtutor }\end{array}$} & $\begin{array}{c}\text { Practicar las } \\
\text { competencias } \\
\text { profesionales relacionadas } \\
\text { con la orientación. }\end{array}$ & $\begin{array}{c}\text { Optimizar el proceso } \\
\text { de adaptación a la } \\
\text { vida universitaria y } \\
\text { laboral. }\end{array}$ & $\begin{array}{c}\text { Desde } 2012 \text { hasta la } \\
\text { actualidad }\end{array}$ & $\begin{array}{l}\text { Facultad de } \\
\text { Educación. } \\
\text { UCM }\end{array}$ \\
\hline & Evaluación & \multicolumn{3}{|c|}{ No existe una evaluación. } \\
\hline \multirow[t]{2}{*}{ Safalud } & $\begin{array}{l}\text { Desarrollar habilidades } \\
\text { sociales y competencias } \\
\text { profesionales. }\end{array}$ & $\begin{array}{l}\text { Conocer los hábitos } \\
\text { alimenticios más } \\
\text { adecuados }\end{array}$ & Desde 2011 & $\begin{array}{l}\text { Colegio } \\
\text { Sagrada Familia } \\
\text { (Valladolid) }\end{array}$ \\
\hline & Evaluación & \multicolumn{3}{|c|}{ No se detalla. } \\
\hline \multirow[t]{2}{*}{ JovexJove } & $\begin{array}{l}\text { Acercar la realidad } \\
\text { del futuro profesional } \\
\text { mediante el contacto } \\
\text { directo }\end{array}$ & $\begin{array}{l}\text { Lograr un apoyo } \\
\text { más directo e } \\
\text { individualizado a los } \\
\text { alumnos con mayores } \\
\text { necesidades. }\end{array}$ & $\begin{array}{c}\text { 2010/2011 hasta la } \\
\text { actualidad }\end{array}$ & $\begin{array}{l}\text { Instituto } \\
\text { La Plana } \\
\text { y Sagrado } \\
\text { Corazón }\end{array}$ \\
\hline & Evaluación & \multicolumn{3}{|c|}{ No se detalla. } \\
\hline
\end{tabular}

Fuente: elaboración propia

En la Figura 3 se detallan, como se ha podido comprobar, diferentes características del ApS queadoptan diversos perfilesen cada experiencia. Respecto a los objetivos, una actividad de Aprendizaje-Servicio se caracteriza por poseer unas metas para los participantes que van más allá de un servicio voluntario a la comunidad. En el ApS se plantean también objetivos relevantes para el aprendizaje académico de ambos grupos.

"Las propuestas de APS combinan estos dos elementos: aprendizajes de contenidos, es decir, adquisición de recursos, por un lado, y entrenamiento en la disponibilidad por movilizarlos en contextos reales, por otro." (Martínez, 2010:20). Por lo tanto, para entender una experiencia realizada en la comunidad como ApS, debe contener unos objetivos orientados a las competencias sociales y ubicadas en el contexto. Por ello, comenzando el análisis por los objetivos dirigidos al aprendizaje de los sujetos provenientes de la universidad, se plantean metas relacionadas con las habilidades sociales, personales o cívicas. También existe un objetivo para los estudiantes universitarios 
que aporta a estos un acercamiento a la realidad laboral, elemento básico de las experiencias de ApS. A partir de los objetivos planteados para dichos sujetos, se puede elaborar una clasificación de los objetivos del aprendizaje-servicio que los distribuye como sigue:

- Desarrollo de competencias sociales y personales:

- Desarrollar capacidad y habilidades para hablar en público.

- Motivar y gestionar grupos.

- Desarrollar habilidades de empatía y responsabilidad

- Desarrollar habilidades personales, relacionales, sociales y cívicas.

- Desarrollar habilidades sociales

- Mejorar la paciencia, empatía.

- Saber hacerse respetar.

- Elaborar diseños y desarrollar proyectos.

- Desarrollo de competencias laborales y acercamiento a la realidad profesional.

- Desarrollar competencias profesionales.

- Acercar al alumno al mundo profesional con niños y jóvenes.

- Practicar las competencias profesionales de orientación.

- Acercar a la realidad del futuro profesional.

- Comprender situaciones de vida en hospitales.

- Desarrollar competencias de la investigación cualitativa.

Como se puede observar, la totalidad de los blogs de experiencias ApS hacen referencia al desarrollo de habilidades sociales o cívicas de los estudiantes universitarios. $Y$ en un alto porcentaje, también hacen referencia a las competencias profesionales y acercamiento a la realidad profesional.

Por otro lado, se encuentran los objetivos relacionados con los otros sujetos, es decir, aquellos que reciben el aprendizaje de los estudiantes universitarios. Dichos sujetos se encuentran, generalmente, en el contexto donde se lleva a cabo la experiencia.

En este caso, los verbos que componen los objetivos son diferentes a los descritos anteriormente y se orientan a otros ámbitos de actuación. Estos objetivos se pueden clasificar en dos áreas:

- Comunicación

- Aumentar el interés y valoración de la expresión oral

- Desarrollar capacidades comunicativas

- Desarrollar habilidades sociales

- Mejorar comunicación oral

- Mejorar capacidades lectoras 
- Acciones en la comunidad

- Lograr un apoyo a los alumnos con mayores necesidades

- Compensar el grave impacto del ingreso hospitalario

- Empoderar su papel en la comunidad

- Reivindicar sus derechos como ciudadanos

- Optimizar el proceso de adaptación a la vida universitaria

- Conocer los hábitos alimenticios

En este caso, tenemos dos áreas muy diferenciadas. Por un lado, se trabajan competencias de comunicación con los otros. Esta área se encuentra presente también en los estudiantes universitarios, sin embargo, el énfasis en estos objetivos se encuentra en la capacidad de comunicación frente al desarrollo de habilidades sociales que se pretende en los estudiantes. Por otro lado, tenemos objetivos relacionados con acciones hacia la comunidad donde se encuentran estos sujetos y que se trabajan a travésde verbos como empoderar, compensar, reivindicar..., que son verbos relacionados con la mejora de las necesidades sociales existentes en estos sujetos.

Con respecto a la temporalidad, en los blogs no se concreta la duración de las actividades, aunque sí se indica o bien desde la fecha en que se viene realizando, o la duración en meses dentro del curso académico. Sobre la fecha de elaboración/fecha de inicio de la experiencia, su duración y actualización, los datos son los siguientes:

- El Taller Comunica comenzó en 2011/2012 y sigue en la actualidad (las noticias colgadas en el blog así lo demuestran).

- El Proyecto ADAMS se inició en 2011, pero la inactividad del blog hace presuponer que no continua en la actualidad.

- El Proyecto PEINAS empezó en 2010 y las noticias publicadas en el blog indican que sigue actualmente.

- Amigos y Amigas de la Lectura comenzó en 2007/2008 pero aparentemente (por la información que aparece en el blog) no continúa en la actualidad.

- Jardín Miguel Martí i Pol se inició en 2005 pero no hay actualizaciones en el blog desde el año 2014.

- El Proyecto SOU-esTUtutor se realiza desde 2012 y continua en la actualidad.

- Safalud comenzó en 2011 y continua en la actualidad.

- JovexJove empezó en el curso 2010/2011 pero no se percibe continuidad a día de hoy.

Todas las experiencias se han comenzado a realizar, como se ha podido comprobar, entre 2005 y 2011, y sólo el 50\% de ellas continúa teniendo actividad en la actualidad. Este dato se ha podido extraer de la sección de noticias o actualidad de cada uno de los blogs. Sin embargo, no se disponen de evidencias que permitan confirmar que las actividades se continúen haciendo pero no se actualicen en el blog. 
En lo que se refiere al lugar donde se llevan a cabo la acción, éste sí se precisa, incluyendo en algunas ocasiones fotografías.

Sobre la evaluación, no todos los blogs incluyen información. Los blogs que incluyen referencias a la evaluación hablan de técnicas y criterios diversos entre los que figuran la participación y la elaboración de un escrito sobre la experiencia (a través de un diario, memoria o portafolio). Uno de ellos va más allá incluyendo un cuestionario de autoevaluación mediante el que valorar la experiencia de ApS.

Por último, en la figura 4, se ha querido anotar las direcciones web de las que se ha extraído la información de las diversas experiencias y proyectos de ApS que se han analizado en estas páginas.

FIGURA 4: DIRECCIONES DE LOS BLOG DE EXPERIENCIAS APS

\begin{tabular}{|c|c|}
\hline Taller Comunica & https://apscomunicacioenpublic.wordpress.com/taller-comunica/ \\
\hline Proyecto ADAMS & http://medikuntzakoikasleak.com/2011/12/04/que-es-el-proyecto-ulises/ \\
\hline Proyecto PEINAS & http://peinasusc.blogspot.com.es/2015/05/plurales-caldas-de-reis.html \\
\hline $\begin{array}{l}\text { Amigos y amigas } \\
\text { de la lectura }\end{array}$ & http://www.aprenentatgeservei.cat/index.php?cm=06\&idC=1008 \\
\hline $\begin{array}{l}\text { Jardín Miguel Martí } \\
\text { i Pol }\end{array}$ & http://jardimiquelmartipol.blogspot.com.es \\
\hline $\begin{array}{l}\text { Proyecto SOU- } \\
\text { esTUtutor }\end{array}$ & https://edusou.wordpress.com \\
\hline Safalud & https://safalud.wordpress.com/¿que-es-safalud/ \\
\hline JovexJove & $\begin{array}{l}\text { https://www.vic.cat/viure-a-vic/educacio/projectes-de-ciutat/ } \\
\text { escola-i-familia/jove-x-joves }\end{array}$ \\
\hline
\end{tabular}

Fuente: elaboración propia

Tras el análisis de la información extraída de los blogs, se puede sostener que esta información no es completa en todos los casos. Tal y como se describía unas páginas atrás, siguiendo la propuesta de Torres-Salinas y Cabezas-Clavijo (2008), sobre el uso de los blogs como tablón de anuncios y repositorio personal o colectivo, se puede afirmar que, en lo referido a las experiencias de aprendizaje-servicio en la universidad española, no se logra este objetivo de aumentar la visibilidad e impacto de los contenidos, ya que no se encuentran desarrollados en su totalidad ni siquiera en aquellos aspectos necesarios para comprender el proceso desarrollado y los resultados obtenidos.

Los blogs analizados proporcionan una visión resumida y puntual de las actividades realizadas en cada una de las experiencias, pero no permite visualizar el proceso 
investigativo que se ha llevado a cabo a lo largo de la experiencia y que nos permitiría saber si la acción realizada nos ayuda a alcanzar los objetivos propuestos.

\section{Conclusiones y propuestas}

La respuesta a la pregunta planteada en el título de este trabajo resulta clara tras los resultados presentados. El análisis realizado de la información obtenida a través de los blogs de experiencias universitarias de aprendizaje-servicio revela la escasa información y el limitado conocimiento que se difunde, y se moviliza, a través de este medio. El papel de los blogs es difundir la información más relevante y, se podría añadir, que a través del blog también se pretende motivar al lector en determinadas cuestiones para hacer feedback sobre elementos concretos de lo publicado y para profundizar en los temas a través de otros proyectos o experiencias. La cuestión es si la información que se sube al blog es suficiente para que las personas interesadas en estas experiencias puedan conocer y entender el proceso seguido para su implementación. Más aún, lo interesante sería compartir conocimiento que pudiera ser útil tanto para futuros proyectos como para la reflexión crítica y la elaboración de nuevos modelos teóricos para este tipo de aprendizaje. Sin embargo, cómo se ha podido apreciar tras el estudio presentado, ya no es sólo que la información que se difunde a través de los blogs sea insuficiente, sino que muchas veces tampoco resulta perceptible qué criterios se han utilizado para seleccionar dicha información y si estos criterios han dado prioridad a la utilidad e interés de los posibles lectores del blog.

En el caso que nos ocupa, no se concretan los resultados de las experiencias (por ejemplo, la opinión de los estudiantes universitarios y de los que han recibido el aprendizaje), sólo se realizan vagas conclusiones. Tampoco se hace referencia a los obstáculos encontrados para su puesta en práctica, lo que sería de gran interés para aquellos interesados en realizar experiencias sobre aprendizaje-servicio.

A partir del análisis realizado, se proponen a continuación una serie de sugerencias para, en primer lugar,el aumento de la movilización del conocimiento generado en los proyectos de ApS que se realizan en el ámbito universitario y, en segundo lugar, la mejora de la visibilidad de los blogs construidos para ello.

- Estructura del blog:

En primer lugar es necesario crear un blog siguiendo un orden lógico. Se debe comenzar realizando una descripción de los sujetos que van a intervenir en la experiencia: quiénes somos, de dónde venimos... Tras esto, habría que indicar cuáles son los elementos básicos de la experiencia: contexto, justificación, experiencias previas, objetivos, temporalidad, evaluación, entre otros.

Una vez definida la parte más estática de nuestro proyecto, es necesario mostrar en el blog la puesta en práctica de las actividades a través de imágenes, videos y pequeños 
textos que permitan al lector ver las facilidades y dificultades a las que los organizadores y participantes de la experiencia se han enfrentado. Por otro lado, sería adecuado mostrar un apartado de noticias, donde se actualice la experiencia con las actividades que se realizan cada año, de modo que se pueda saber si es una actividad aislada en un año académico o tiene continuidad en el tiempo.

Por último, sería importante reseñar un medio de contacto por el cual, los interesados en participar o en conocer más acerca de la experiencia, puedan ponerse en contacto con los organizadores. Esto puede ayudar a la difusión y realización de la práctica en más centros o instituciones y a comparar los resultados entre las actividades de ApS realizadas.

- Situación del blog:

Otra propuesta para la correcta difusión de las experiencias es la relacionada con la publicidad que se le da al blog. Escribir un blog y no posicionarlo correctamente en internet no ayuda a que otras personas puedan leer acerca del proceso y de los resultados obtenidos. Por eso, resulta fundamental que los blogs sobre ApS se cuelguen en la Red Española de Aprendizaje-Servicio lo que, en el caso de la muestra seleccionada, no en todos los casos se ha hecho así. La realización de un blog se hace con la intención de llegar al mayor número de personas, de aquí que no sólo sea necesario crear el blog sino que es importante colgarlo en aquellos lugares donde acceden los interesados en cada especialidad.

- Uso de redes sociales como apoyo:

Otra propuesta para mejorar aún más la visibilidad de nuestras experiencias es el apoyo del blog a través de redes sociales como Facebook o Twitter. Estas plataformas se caracterizan por poseer la mayor red de contactos con la que se puede contar. Por ello sería conveniente anunciarlo a través de estas plataformas y mostrar en ellas los diferentes apartados del blog, de modo que el potencial público pueda acceder a este a través de las redes sociales.

La mejora de la visibilidad de los blogs puede ayudar a la proliferación de más experiencias como estas, las cuales pueden constituir un gran aliado para el docente en el aula. Además,el poder contar con una mayor muestra de alumnado participante en una experiencia similar, permite realizar investigaciones que nos indiquen más certeramente los beneficios o no de su realización.

El uso de los blogs es provechoso para la práctica docente, ya que, además de las diversas ventajas mencionadas, también tiene otra relacionada con la comunicación. Los blogs permiten al docente estar en contacto con sus estudiantes y con la comunidad de aprendizaje afín a su área de especialización. Los blogs son, pues, una herramienta con un enorme potencial para los docentes y la investigación que realizan 
dentro y fuera del aula. De su creación, construcción y buen uso, dependerá la amplitud y diversidad de sus beneficios.

\section{Referencias}

Aramburuzabala, P; Cerrillo, R y Tello, I. (2015). Aprendizaje-servicio: una propuesta metodológica para la introducción de la sostenibilidad curricular en la universidad. Profesorado. Revista de currículum y formación del profesorado, 19(1), 78-95

Deeley, S. J. (2016). El Aprendizaje-Servicio en educación superior. Teoría, práctica y perspectiva crítica. Madrid: Narcea.

Garrido, A. (2003). El aprendizaje como identidad de participación en la práctica de unacomunidad virtual. Barcelona: UOC.

Martínez, M. (2010). Aprendizaje servicio y construcción de ciudadanía activa en la universidad: la dimensión social y cívica de los aprendizajes académicos. In M. Martínez (ed.),Aprendizaje servicio y responsabilidad social de las universidades (pp.11-26). Barcelona: Octaedro/ICE UB.

Orellana López, D.M. y Sánchez Gómez, M.C. (2006). Técnicas de recolección de datos en entornos virtuales más usadas en la investigación cualitativa. Revista de Investigación Educativa, 24(1), 205222.

Puig, J. M., Batlle, R., Bosch, C., y Palos, J. (2007). Aprendizaje servicio. Educar para la ciudadanía. Barcelona: Octaedro.

Rodríguez-Gallego, M. R. (2014). El aprendizaje-servicio como estrategia metodológica en la universidad. Revista Complutense de Educación, 25 (1), 95-113.

Rubio, L.; Prats, E. y Gómez, L. (coord.) (2013). Universidad y sociedad. Experiencias de aprendizaje servicio en la universidad. Barcelona: Universitat de Barcelona - Institut de Ciències de l'Educació.

Santos, M. A., Sotelino, A. y Lorenzo, M. (2015). Aprendizaje-servicio y misión cívica de la universidad. Una propuesta de desarrollo. Barcelona: Octaedro.

Torres-Salinas, D., y Cabezas-Clavijo, A. (2008). Los blogs como nuevo medio de comunicación científica. Lecture, III Encuentro Ibérico de Docentes e Investigadores en Información y Documentación, Salamanca [Disponívelem http://eprints.rclis.org/14078/1/Torres-Salinas\%2C_Daniel_y_CabezasClavijo\%2C_Alvaro._Los_blogs_como_nuevo_medio_de_comunicacion_cientifica.pdfconsultado em 10/11/2016].

María R. Belando-Montoro

Doctora en Pedagogía y Profesora Titular de Universidad en el Departamento de Teoría e Historia de la Educación de la Universidad Complutense de Madrid. Es coordinadora del Máster en Estudios Avanzados en Educación Social. Sus líneas de investigación giran alrededor de la formación permanente, la pedagogía social, la gerontología educativa y la enseñanza universitaria. Email: mbelando@ucm.es ORCID: 0000-0001-5730-7896 
María Aranzazu Carrasco Temiño Licenciada en Pedagogía y Máster Universitario en Estudios Avanzados en Pedagogía por la Universidad Complutense de Madrid. Colaboradora honorífica del Departamento de Teoría e Historia de la Educación en la Universidad Complutense de Madrid, donde realiza asimismo estudios de postgrado. Actualmente realiza su labor profesional en el desarrollo de un proyecto de asesoría sociolaboral a centros sociosanitarios siendo responsable de RRHH en el área de formación y selección.

Email: arantxa.carrasco@hotmail.com

ORCID: 0000-0001-5760-8234

Correspondência

María R. Belando-Montoro Departamento de Teoría e Historia de la Educación Facultad de Educación Universidad Complutense de Madrid $\mathrm{c} /$ Rector RoyoVillanova s/n 28040- Madrid (España)

Data de submissão: Dezembro 2016

Data de avaliação: Janeiro 2017

Data de publicação: Março de 2018 